INPLASY

PROTOCOL

To cite: Gupta et al. How effective are devices in the management of

Onychomycosis? A protocol

for a systematic review. Inplasy

protocol 2021110069. doi:

10.37766/inplasy2021.11.0069

Received: 17 November 2021

Published: 17 November 2021

Corresponding author:

Aditya Gupta

agupta@mediproberesearch.com

Author Affiliation:

Mediprobe Research Inc.

Support: Self-financed.

Review Stage at time of this submission: The review has not yet started.

Conflicts of interest:

None declared.

\section{How effective are devices in the management of Onychomycosis? A protocol for a systematic review}

Gupta, AK¹; Hall, DC2; Simkovich, AJ³.

Review question / Objective: To compile evidence and determine the utility of various device-based treatments for the treatment of onychomycosis, and improve the appearance of afflicted toenails.

Condition being studied: Toenail onychomycosis, also known as tinea unguium.

Information sources: A systematic literature search will be conducted in Scopus, MEDLINE and PubMed. The bibliographies of relevant articles will also be searched to ensure a comprehensive review is presented. Clinical trial registries (such as clinicaltrials.gov) will also be searched for complete, or incomplete trials.

INPLASY registration number: This protocol was registered with the International Platform of Registered Systematic Review and Meta-Analysis Protocols (INPLASY) on 17 November 2021 and was last updated on 17 November 2021 (registration number INPLASY2021110069).

\section{INTRODUCTION}

Review question / Objective: To compile evidence and determine the utility of various device-based treatments for the treatment of onychomycosis, and improve the appearance of afflicted toenails.

Rationale: Onychomycosis is a chronic, fungal infection of the nail, accounting for over half of all nail disorders. Dermatophytes are the primary causal agents, however non-dermatophyte molds, and yeasts are also implicated in this disease. At first, the fungus colonizes the nail plate before reaching the nail bed and matrix. Infection of toenails is more common, likely due to the warm, damp environment created by feet clad in socks and footwear. Common treatments include oral and topical antifungal medications. Despite their moderate to high efficacy, clinicians and patients are concerned of reported adverse events and potential for 
hepatotoxicity and drug-drug interactions. In recent years, device-based treatments have gained popularity for the treatment option for onychomycosis. Non-drug treatments, are evaluated differently by regulatory agencies such as the United States Food and Drug Administration (USFDA). These devices are measured by their ability to improve the appearance of afflicted nails. For example, antifungals are evaluated by the FDA for the treatment of toenail onychomycosis by reporting medical endpoints such as complete and mycological cure. Some devices, such as specific lasers are FDA-approved only for "temporary increase of clear nail in patients with onychomycosis". Other devices used in onychomycosis treatment include photodynamic therapy, ultrasound, nonthermal plasma, iontophoresis, and the physical creation of microchannels or holes in the nail plate. In most cases, these devices are used in concert with a topical agent, proximal to the infection site. Our objective is to summarize the role of devices in the management of onychomycosis as there is limited data in the literature regarding their efficacy and safety. Due to the paucity of evidence from randomized controlled trials and lack of comprehensive reviews, very few conclusions of these types of treatments have been made. We propose to summarize and critically review the available body of evidence to aid in the evidence based decision making process of clinicians and their patients.

Condition being studied: Toenail onychomycosis, also known as tinea unguium.

\section{METHODS}

Search strategy: Six systematic searches will be conducted (one for each intervention). For each, we will search Scopus and MEDLINE databases. Search Phrases will include: ((onychomycosis OR superficial mycoses OR fungal infection OR tinea unguium) AND (laser*))

((onychomycosis OR superficial mycoses OR fungal infection OR tinea unguium) AND (Iontophoresis)) ((onychomycosis OR superficial mycoses OR fungal infection OR tinea unguium) AND (Drilling))

((onychomycosis OR superficial mycoses OR fungal infection OR tinea unguium) AND (Plasma))

((onychomycosis OR superficial mycoses OR fungal infection OR tinea unguium) AND (Ultrasound))

((onychomycosis OR superficial mycoses OR fungal infection OR tinea unguium) AND (Microwave)).

Participant or population: The patient population of interest is men and women clinically and mycologically (microscopy, fungal culture) diagnosed with toenail onychomycosis. Patients from any ethnicity/race or age will be included.

Intervention: Medical devices such as lasers, photodynamic therapy, iontophoresis, plasma, ultrasound, microwaves and the creation of microchannels for toenail onychomycosis will be investigated.

Comparator: (1) no treatment (i.e., nothing) (2) $\mathrm{placebo} / \mathrm{mock} / \mathrm{sham}$ treatment(3) Conventional antifungal treatment (oral, topical, or a combination of both).

Study designs to be included: Data for the proposed study will be gathered from in vivo trials including randomized controlled trials, pilot single-arm trials and observational studies (ie. case reports).

Eligibility criteria: When available, in vivo studies evaluating a single toenail were preferred. Monotherapy are required for inclusion of in vivo or in vitro studies.

Information sources: A systematic literature search will be conducted in Scopus, MEDLINE and PubMed. The bibliographies of relevant articles will also be searched to ensure a comprehensive review is presented. Clinical trial registries (such as clinicaltrials.gov) will also be searched for complete, or incomplete trials. 
Main outcome(s): Main outcomes will include the clinical and mycological cure rates of onychomycosis treated with these devices.

Data management: Data will be organized into spreadsheets.

Quality assessment / Risk of bias analysis: The quality of evidence across studies will be evaluated using the Grading of Recommendations, Assessment, Development and Evaluations (GRADE) framework.

Strategy of data synthesis: The goal of this project is to conduct a systematic review.

Subgroup analysis: None.

Sensitivity analysis: None.

Language: Only publications in the English language will be included.

Country(ies) involved: Canada.

Keywords: Onychomycosis; superficial mycoses; Tinea unguium; iontophoresis; nail drilling; photodynamic therapy; cold plasma; non-thermal plasma; ultrasound; photodynamic therapy.

Contributions of each author:

Author 1 - Aditya Gupta - Study conceptualization, will oversee the search process, make final decisions when other authors have conflict for article inclusion (if any), edit and review manuscript throughout its completion.

Email: agupta@mediproberesearch.com

Author 2 - Deanna Hall - Partake in initial searching, make decisions of exclusion of articles based on title, abstract, or contents. Assist in writing and editing of manuscript.

Email: dhall@mediproberesearch.com

Author 3 - Aaron Simkovich - Partake in initial searching, make decisions of exclusion of articles based on title, abstract, or contents, created illustrative figures, primary writer and editor of this manuscript.

Email: asimkovich@mediproberesearch.com 